\title{
I-BET726 suppresses human skin squamous cell carcinoma cell growth in vitro and in vivo
}

\author{
Zhengjun Liu', Ping Li ${ }^{2}$, Yong-qiang Yang $\mathbb{0}^{3}$, Shang Cai ${ }^{3}$, Xiangwei Lin ${ }^{1}$, Min-bin Chen $^{2}$ and Hailei Guo ${ }^{1}$
}

\begin{abstract}
Bromodomain-containing protein 4 (BRD4) is a potential therapeutic target of skin squamous cell carcinoma (SCC). IBET726 is a novel BRD4 inhibitor. Its potential effect in skin SCC cells was tested in the present study. We show that IBET726 potently inhibited survival, proliferation, cell cycle progression, and migration in established (A431/SCC-9/SCC12/SCC-13 lines) and primary human skin SCC cells. I-BET726 induced significant apoptosis activation in skin SCC cells. It was more efficient in inhibiting skin SCC cells than known BRD4 inhibitors (JQ1, CPI203, and AZD5153). I-BET726 not only downregulated BRD4-regulated proteins (C-Myc, BCl-2, and cyclin D1), but also inhibited sphingosine kinase 1 (SphK1) and Akt signalings in SCC cells. Restoring Akt activation, by a constitutively active S473D mutant Akt1 ("caAkt1"), partially inhibited I-BET726-induced cytotoxicity in A431 cells. In vivo, I-BET726 oral administration potently inhibited A431 xenograft growth in severe combined immunodeficient mice. Downregulation of BRD4-regulated proteins and inhibition of the SphK1-Akt signaling were detected in I-BET726-treated A431 xenograft tumor tissues. Together, I-BET726 inhibits skin SCC cell growth in vitro and in vivo.
\end{abstract}

\section{Introduction}

Skin squamous cell carcinoma (SCC) causes significant cancer-related human mortalities ${ }^{1-3}$. The prognosis for the advanced, metastatic, and recurrent skin SCC is poor $^{1-3}$. Molecularly targeted therapies are desperately needed for better skin SCC therapy ${ }^{1-3}$.

Bromodomain and extraterminal (BET) family proteins have emerged as exciting and novel therapeutic proteins for cancer ${ }^{4,5}$. Bromodomain-containing protein 4 (BRD4) is the most studied and a primary member of BET family. BRD4 overexpression and/or hyperactivation is associated with tumorigenesis of hematological and solid tumors ${ }^{4,5}$. BRD4 regulates transcription elongation of key genes of

Correspondence: Min-bin Chen (cmb1981@163.com) or

Hailei Guo (zxkguohl@163.com)

${ }^{1}$ Department of Burn and Plastic Surgery, The First Affiliated Hospital of

Wenzhou Medical University, Wenzhou, China

${ }^{2}$ Department of Radiotherapy and Oncology, Affiliated Kunshan Hospital of

Jiangsu University, Kunshan, China

Full list of author information is available at the end of the article

These authors contributed equally: Zhengjun Liu, Ping Li, Yong-qiang Yang,

Shang Cai

Edited by M. Agostini cell proliferation, cell cycle progression, and apoptosis, including $c-M y c, B c l-2$, and cyclin $D 1^{5}$. BRD4 inhibitors have been tested in a number of preclinical cancer studies, showing promising anticancer outcomes. Our previous study has shown that overexpression of BRD4 in human skin SCC cells can promote cell growth in vitro and in vivo ${ }^{6}$. BRD4 shRNA or knockout (by CRISPR/Cas9 method) potently inhibited skin SCC cell proliferation. Reversely, forced overexpression of BRD4 facilitated skin SCC cell proliferation ${ }^{6}$. These results suggest that targeting BRD4 could be a novel and efficient strategy against skin SCC cells.

I-BET726 (GSK1324726A) is a novel and potent inhibitor of BET family proteins, showing high affinity binding to BRD47. I-BET726 competes with tetra-acetylated histones for binding to the bromodomain of $\mathrm{BRD} 4{ }^{7}$. IBET726 exhibited over 1000-fold selectivity of BRD4 than the bromodomain-containing homologs ${ }^{7}$. The compound displayed favorable physicochemical and pharmacokinetic properties along with acceptable safety profiles, suitable for potential clinical development ${ }^{7}$. Its potential activity in human skin SCC cells has not been studied.

\section{(c) The Author(s) 2020}

(c) (i) Open Access This article is licensed under a Creative Commons Attribution 4.0 International License, which permits use, sharing, adaptation, distribution and reproduction cc) in any medium or format, as long as you give appropriate credit to the original author(s) and the source, provide a link to the Creative Commons license, and indicate if changes were made. The images or other third party material in this article are included in the article's Creative Commons license, unless indicated otherwise in a credit line to the material. If material is not included in the article's Creative Commons license and your intended use is not permitted by statutory regulation or exceeds the permitted use, you will need to obtain permission directly from the copyright holder. To view a copy of this license, visit http://creativecommons.org/licenses/by/4.0/. 


\section{Materials and methods}

\section{Chemicals and reagents}

I-BET726 was purchased from Adooq (Shanghai, China). MTT, AZD5153, PD98059 and LY294002, JQ1 and CPI203 were purchased from Sigma (Shanghai, China). Z-DEVD-fmk, Z-LEHD-fmk, and Z-VAD-fmk were provided by Calbiochem (La Jolla, CA). Antibodies for c-Myc (\#9402), Cyclin D1 (\#2922), BRD4 (\#13440), Bcl-2 (\#15707), SphK1 (\#12071), phosphorylated ("p")Akt (Ser-473) (\#9271), Akt1/2 (\#9272), p-p44/42 MAPK (Erk1/2) (\#9101) and Erk1/2 (\#9102), cleaved-caspase-3 (\#9664), cleaved-caspase-8 (\#9496), cleaved-caspase-9 (\#20750), cleaved-poly (ADP-ribose) polymerase (PARP) (\#5625), $\beta$-tubulin (\#15115) and $\beta$-actin (\#3700) were purchased from Cell Signaling Tech (Beverly, MA).

\section{Cell culture}

The established skin SCC cell lines, A431, SCC-9, SCC12, and SCC-13, were purchased from the Cell Bank of Chinese Academy of Science (Shanghai, China). The cell culture procedure was described early ${ }^{6}$. Cells were subjected to mycoplasma and microbial contamination examination every 3-4 months. Authentication by short tandem repeat profiling, population doubling time, and morphology were routinely confirmed as well to verify the genotype. The primary human skin SCC cells were provided by Dr. Wang ${ }^{8}$. Cells were derived from two writteninformed consent SCC patient ("C1/C2", with PTEN depletion and p53-null) and cultured as described previously $^{6}$. Cultures of primary human skin keratinocytes and fibroblasts were reported previously ${ }^{6,9}$. The protocols of using primary human cells were conducted to accordance with the Declaration of Helsinki, with approval by the Ethics Board of Wenzhou Medical University.

\section{MTT assay}

Cells were seeded onto 96-well plates $\left(5 \times 10^{3}\right.$ cells per well). After I-BET726 treatment, cell viability was tested by MTT dye assay. MTT optical densities were measured at $550 \mathrm{~nm}$ using a microplate reader.

\section{Soft agar colony formation}

Following I-BET726 treatment, A431 cells were placed onto $10-\mathrm{cm}$ dishes $\left(2 \times 10^{4} \text { cells per treatment }\right)^{10}$. IBET726 medium was renewed every two days. After 10 days, the number of A431 colonies was counted.

\section{Hoechst-33342 staining of apoptotic cells}

Cells were plated onto 24 -well plates $\left(2 \times 10^{4}\right.$ cells per well). Following the treatments, cells were stained with Hoechst-33342 (Sigma). Nuclei with intensified Hoechst33342 condensation or fragmentation were labeled as nuclei of apoptotic cells. The nuclei of non-apoptotic cells showed the faint delicate Hoechst-33342 staining. At least
300 cells from five random-selected views (1:100 under microscope) were included to calculate the apoptotic nuclei ratio.

\section{BrdU assay}

Cells were seeded onto 96 -well plates $\left(5 \times 10^{3}\right.$ cells per well), treated with I-BET726 in the presence of BrdU $(10 \mu \mathrm{M}$, Cell Signaling Tech). BrdU incorporation was examined by an ELISA kit (Cell Signaling Tech). BrdU enzyme-linked immunosorbent assay (ELISA) OD values at $405 \mathrm{~nm}$ were recorded.

\section{EdU (5-ethynyl-20-deoxyuridine) assay}

Cells were initially seeded into six-well plates (at 100,000 cells in each well). After the applied treatment, EdU Apollo-567 assay kit (RiboBio, Guangzhou, China) ${ }^{11,12}$ was applied to examine and quantify cell proliferation. The nuclear EdU and DAPI staining were visualized through a fluorescent microscope. Five random views with total $800-1000$ cells of each treatment were included to calculate EdU/DAPI ratio.

\section{In vitro cell migration assay}

Skin SCC cells with the applied treatments were initially seeded on the "Transwell" upper chamber (BD Biosciences, Shanghai, China), at a density of 10,000 cells in $300 \mu \mathrm{L}$ serum-free medium (each chamber) ${ }^{13}$. FBScontaining complete medium was added to the lower chamber surface. After incubation for $48 \mathrm{~h}$ the migrated cells on the lower surface were stained and counted. Five repeated views of each condition were included to calculate the average number of migrated cells.

\section{Cell cycle assay}

Cells with I-BET726 treatment were fixed and stained with propidium iodide (PI, $10 \mu \mathrm{g} / \mathrm{mL}$, Invitrogen) and RNase $(100 \mu \mathrm{g} / \mathrm{mL}$, Invitrogen). A flow cytometer (BD Biosciences, Franklin Lakes, NJ) was employed to analyze DNA content. Cell cycle distribution percentages (G0/1-, $\mathrm{S}$ - and G2/M-phases) were recorded.

\section{Caspase activity assay}

The cytosolic protein extracts ( $30 \mu \mathrm{g}$ per treatment) were incubated with the 7-amino-4-trifluoromethylcoumarin (AFC)-conjugated caspase $(-3 /-8 /-9)$ substrates $(10 \mu \mathrm{g} /$ $\mathrm{mL}$ each) in the caspase assay buffer. AFC intensities were tested by Infinite 200 PRO reader at $400 \mathrm{~nm}$ excitation and $505 \mathrm{~nm}$ emission.

\section{Annexin V assay}

In brief, following the treatments, cells were stained with Annexin V-FITC $(10 \mu \mathrm{g} / \mathrm{mL})$ and PI $(10 \mu \mathrm{g} / \mathrm{mL})$ (Sigma). Afterwards, cells were detected via fluorescenceactivated cell sorting on a FACSCalibur machine (BD 
Biosciences). Annexin $\mathrm{V}^{+/+}$cells were labeled as apoptotic cells.

\section{Lactate dehydrogenase (LDH) assay of cell death}

In brief, following the indicated treatment, medium LDH was collected and tested by a two-step LDH detection kit (Promega, Shanghai, China), and normalized to total LDH contents.

\section{Western blotting assay}

As described ${ }^{9}$, the quantified protein lysates $(30 \mu \mathrm{g}$ proteins per treatment) were separated by $10 \%$ sodium dodecyl sulphate-polyacrylamide gel electrophoresis, transferred to the PVDF blots (Biyuntian). After blocking, the blots were immunoblotted with applied primary antibodies, followed by incubation with HRP-conjugated secondary antibodies. The enhanced chemiluminescence reagents (Pierce) were added to visualize the targeted protein bands. Total gray of each band was quantified by the ImageJ software ${ }^{14}$.

\section{Sphingosine kinase 1 (SphK1) activity assay}

SphK1 activity was detected by a previously described protocol $^{15,16}$. SphK1 activities were valued as pmol/hour/ g protein, normalized to the control.

\section{Ceramide assay}

Using a previously described protocol ${ }^{17}$, the cellular ceramide content was analyzed, and its value was expressed as fmol by nmol of phospholipid. Ceramide content in I-BET726-treated cells was always normalized to that of untreated control cells.

\section{Constitutively active mutant Akt1}

The purified constitutively active S473D mutant Akt1 ("caAkt1") recombinant adenovirus and empty vectors recombinant adenovirus (Ad-GFP) were both provided by Dr. Cao at Soochow University ${ }^{18}$. Cells were initially seeded into six-well plates (at 100,000 cells in each well). The adenovirus was added to cultured cells for $48 \mathrm{~h}$. The stable cell lines were established via selection for 4-5 passages. For virus infection, cells were cultured in polybrene-containing medium.

\section{BRD4 knockout (KO)}

The CRISPR/Cas9 BRD4-KO plasmid (sc-400519-KO2; Santa Cruz Biotechnology, Shanghai, China) was transfected to cultured A431 cells via the Lipofectamine 2000 protocol (Invitrogen, Shanghai, China). The transfected cells were further selected with puromycin after 4-5 passages, and two stable cell lines were established ("BRD4-KO-sL1/2"). Control cells were treated with an empty vector with Cas9 control construct ("Cas9-C"; Santa Cruz Biotechnology). BRD4 expression in stable cells was verified by western blotting.

\section{Xenograft assay}

As described ${ }^{6,9}$ A431 cells $\left(6 \times 10^{6}\right.$ cells in $200 \mu \mathrm{L}$ matrigel/serum-free medium, for each mouse) were inoculated via subcutaneous (s.c.) injection to the right flanks of the severe combined immunodeficient (SCID) mice (female, 6-7 weeks old). Within 18-20 days the volume of each tumor was close to $100 \mathrm{~mm}^{3}$, and the A431 tumor xenografts were established. SCID mice were randomly assigned into three groups (10 mice per group). Every 5 days tumor volumes and mice body weights were recorded. All animal studies were performed according to the standards of ethical treatment and IACUC of Wenzhou Medical University. The protocols were approved by the Ethics Committee (2016-R311) of Wenzhou Medical University.

\section{Statistical analysis}

The investigators were blinded to the group allocation during all experiments. In vitro experiments in this study were repeated at least three times. Data were presented as mean \pm standard deviation (SD). Statistics were analyzed by one-way analysis of variance by SPSS software (21.0, Chicago, CA). To compare difference between two specific groups, a two tailed $t$ test was applied (Excel 2007). $p$ values $<0.05$ were considered statistically different. All the protocols of this study were approved by Ethics Committee of Wenzhou Medical University.

\section{Results}

I-BET726 inhibits human skin SCC cell viability, proliferation, cell cycle progression, and migration

A431 SCC cells were treated with I-BET726 at gradually increased concentrations (5-100 nM). MTT assay results, in Fig. 1a, show that I-BET726, in a concentrationdependent manner, potently inhibited A431 cell viability. I-BET726 also displayed a time-dependent response in inhibiting A431 cell viability (Fig. 1a). The IC-50 of IBET726 was close to $10-50$ nм (72 h, Fig. 1a). A431 cell proliferation was analyzed by soft agar colony formation assay and BrdU incorporation ELISA assay. As demonstrated, I-BET726 dose-dependently decreased the number of A431 cell colonies (Fig. 1b) and BrdU ELISA OD (Fig. 1c), indicating an antiproliferative activity by IBET726. EdU incorporation assay results, Fig. 1d, demonstrated that I-BET726 treatment $(50 \mathrm{~nm}, 48 \mathrm{~h})$ potently decreased EdU ratio in A431 cells, further confirming proliferation inhibition. In addition, when analyzing cell cycle progression, we show that I-BET726 (50 nM) disrupted cell cycle progression, causing G1-S arrest in A431 cells (Fig. 1e). By counting the number of the migrated cells in the "Transwell" assay, we show that 


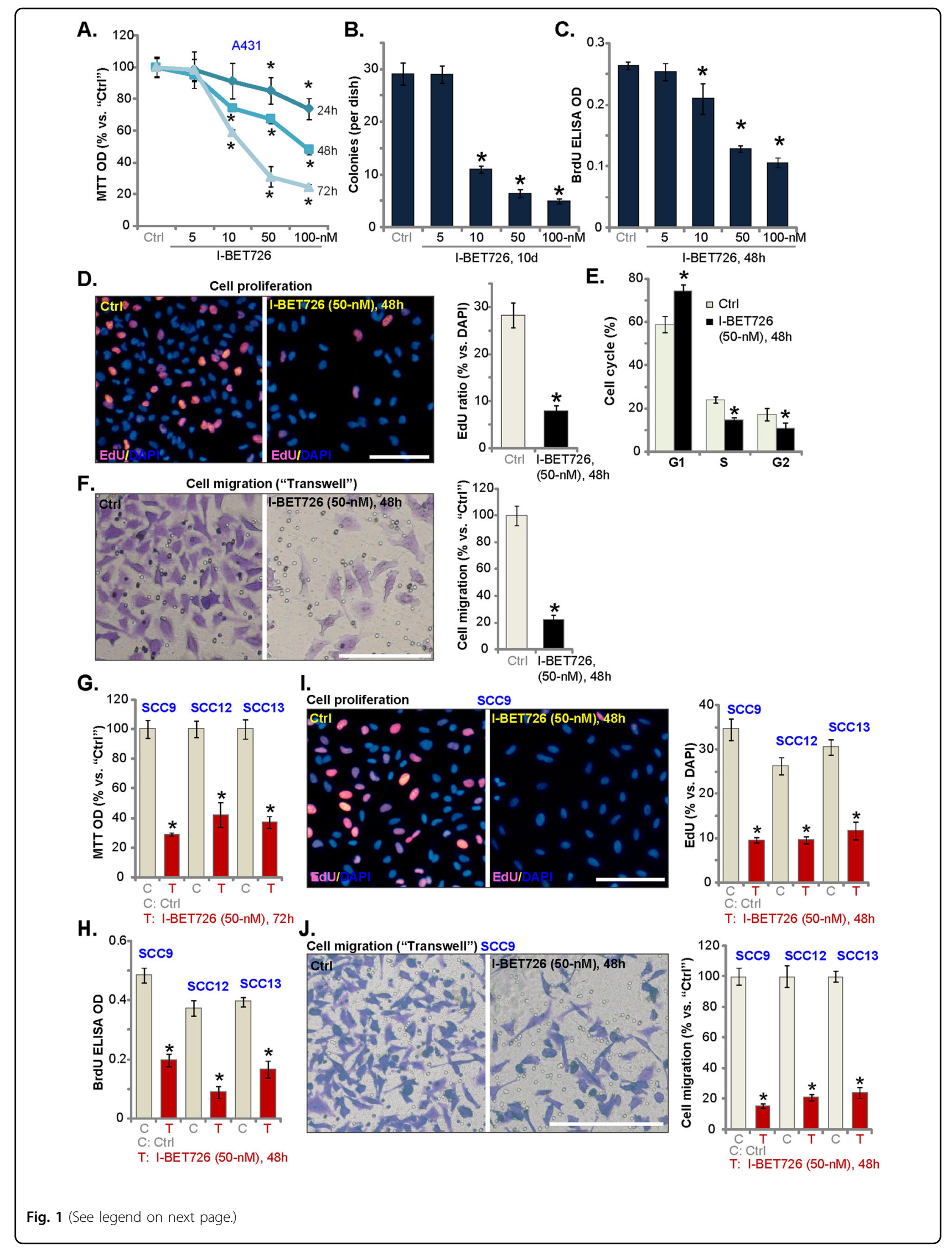


(see figure on previous page)

Fig. 1 I-BET726 inhibits survival, proliferation, cell cycle progression, and migration in established SCC cells. A431 cells a-f SCC-9, SCC-12, or SCC-13 cells $\mathbf{~ g - j}$ were left untreated ("Ctrl", same for all Figures), or treated with I-BET726 (5-100 nM), cells were further cultured in I-BET726-containing medium for indicated time periods, cell viability $\mathbf{a}, \mathbf{g}$, proliferation $(\mathbf{b}-\mathbf{d}, \mathbf{h}, \mathbf{i})$, cell migration $\mathbf{f}, \mathbf{j}$, and cell cycle progression $\mathbf{e}$ were tested by the appropriate assays. Data were presented as mean \pm standard deviation (SD) (Same for all Figures). $n=5$ stands for five replicate wells/dishes (Same for all Figures). ${ }^{*} p$ $<0.05$ vs. "Ctrl" group. All in vitro experiments in this study were repeated three times with similar results obtained. Bar $=100 \mu \mathrm{m} \mathbf{d}, \mathbf{f}$, i and $\mathbf{j}$.

I-BET726 (50 nM, 24 h) significantly inhibited A431 cell migration in vitro (Fig. 1F).

In other three established SCC cell lines, SCC-9, SCC12, SCC-13, I-BET726 treatment (50 nM, 24-72 h) robustly decreased cell viability (Fig. 1g), BrdU ELISA OD (Fig. 1h) and EdU incorporation (Fig. 1i). Furthermore, "Transwell" assay results, Fig. 1j, demonstrated that IBET726 inhibited in vitro migration of the tested SCC cells.

Next, we tested the potential effect of I-BET726 in the primary human cells. The primary human skin SCC cells, derived from two skin SCC patients ("C1/C2"), were treated I-BET726 (50 nM). As shown, I-BET726 significantly inhibited viability (MTT OD, Fig. 2a), proliferation (BrdU ELISA OD, Fig. 2b) and EdU incorporation (Fig. 2c) of primary SCC cells. On the contrary, it was ineffective in the primary human skin keratinocytes ("Kera") and fibroblasts (Fig. 2a-c), where BRD4 levels are low ${ }^{6}$. Further studies show that the BRD4 inhibitor suppressed migration of primary human SCC cells (Fig. 2d), but being invalid in skin fibroblasts and keratinocytes (Fig. 2d).

We compared the activity of I-BET726 with other known BRD4 inhibitors, including JQ1 $1^{19,20}$, CPI203 ${ }^{6,21}$, and AZD5153 ${ }^{22,23}$. In A431 cells, I-BET726 (50 nM) was significantly more potent in inhibiting cell viability (Fig. $\mathrm{S} 1 \mathrm{~A}$ ) and proliferation (Fig. S1B) than even higher concentrations of JQ1 (500 nM), CPI203 (500 nM), and AZD5153 (100 nM). These results show that I-BET726 potently inhibits survival, proliferation and migration of established and primary human skin SCC cells.

\section{I-BET726 induces human skin SCC cell apoptosis}

Previous studies have demonstrated that BRD4 inhibition or depletion could induce apoptosis activation in human cancer cells ${ }^{24-26}$. The potential effect of I-BET726 on skin SCC cell apoptosis was studied next. As shown, IBET726 dose-dependently increased the activities of caspase- 3 and caspase- 9 in A431 cells (Fig. 3a). Caspase- 8 activity, an indicator of extrinsic apoptosis pathway activation $^{27}$, was unaffected (Fig. 3a). Further, levels of cleaved ("Cle")-caspase-3, cleaved-PARP and cleavedcaspase-9 were increased in I-BET726 (10-100 nM)-treated A431 cells (Fig. 3b), where cleaved-caspase-8 levels were again unaffected (Fig. 3b). BRD4 expression was unchanged by I-BET726 treatment (Fig. 3b). Following I-
BET726 treatment, the percentage of nuclei with condensed/fragmented Hoechst-33342 staining ("apoptotic nuclei") was significantly increased (Fig. 3c). Moreover, IBET726, in a dose-dependent manner, increased the percentage of A431 cells with positive Annexin V staining (Fig. 3d). At lowest concentration (5 $\mathrm{nM})$, it was ineffective on cell apoptosis (Fig. 3a-d). Therefore, I-BET726 dosedependently induced apoptosis activation in A431 cells.

Further experimental results show that terminal deoxynucleotidyl transferase dUTP nick end labeling (TUNEL)-positive nuclei ratio was significantly increased in I-BET726 (50 nM, $48 \mathrm{~h}$ )-treated A431 cells (Fig. 3e). Importantly, I-BET726 (50 nM)-induced apoptosis activation was more potent than other known BRD4 inhibitors: JQ1, CPI203, and AZD5153 (Fig. S1C). Different caspase inhibitors, including the caspase-3 specific inhibitor $\mathrm{z}$ DEVD-fmk, the caspase-9 specific inhibitor z-LEHD-fmk and the pan caspase inhibitor $\mathrm{z}$-VAD-fmk, were utilized. In Fig. 3f, MTT assay results show that pre-treatment (for $30 \mathrm{~min}$ ) with the caspase inhibitors significantly attenuated I-BET726 (50 nM, $72 \mathrm{~h}$ )-induced A431 cell viability reduction. Therefore, apoptosis activation should be the primary reason of I-BET726-induced cytotoxicity in A431 cells. In SCC-9, SCC-12, and SCC-13 cells IBET726 treatment $(50 \mathrm{nM}, 48 \mathrm{~h})$ increased TUNEL staining ratio as well (Fig. 3g).

In the primary skin SCC cells ("C1/C2"), I-BET726 (50 nM) significantly increased the Annexin V-positive cells (Fig. 3h), suggesting apoptosis activation. No significant apoptosis was yet detected in I-BET726-treated human skin keratinocytes and fibroblasts (Fig. 3h). Collectively, these results show that I-BET726 induces apoptosis activation in skin SCC cells.

\section{I-BET726 inhibits BRD4, SphK1, and Akt signalings in human skin SCC cells}

BRD4 recruits and phosphorylates P-TEFb (the positive transcription elongation factor $\mathrm{b}$ ), required for transcription elongation ${ }^{4,5}$. Several BRD4-dependent proteins (including $\mathrm{c}-\mathrm{Myc}, \mathrm{Bcl}-2$, and cyclin D1) are key oncogenes $^{4,5}$. In A431 cells, I-BET726 dose-dependently inhibited $\mathrm{c}-\mathrm{Myc}, \mathrm{Bcl}-2$, and cyclin $\mathrm{D} 1$ protein expression (Fig. 4a). I-BET726 is more potent than other BRD4 inhibitors in inhibiting skin SCC cells (Figs. 1, 2), therefore it is possible that I-BET726 could regulate BRD4indendent pathways. To support our hypothesis, the 


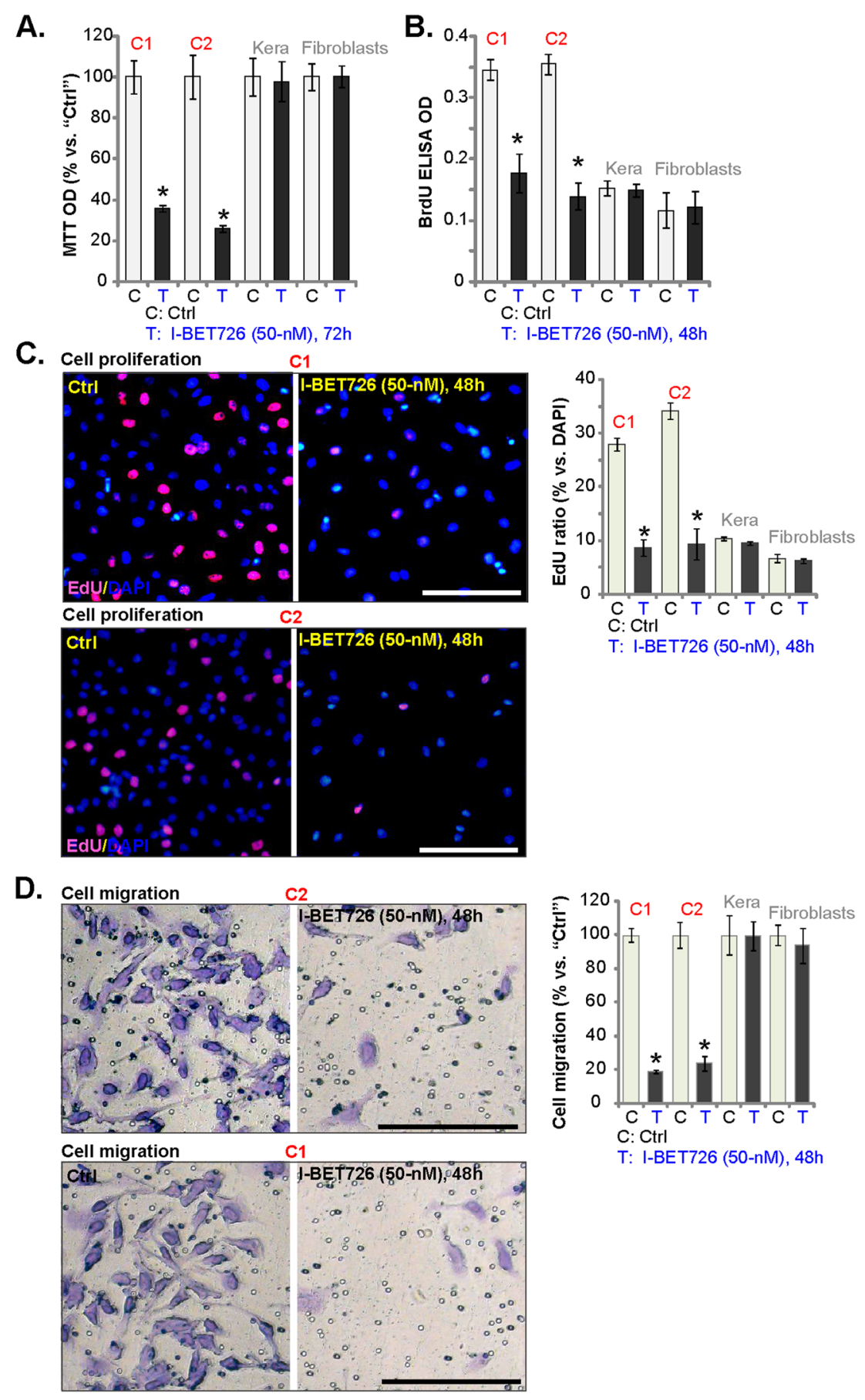

Fig. 2 I-BET726 inhibits survival, proliferation and migration in primary human SCC cells. The primary human skin SCC cell ("C 1 "/" $C 2$ "), human skin keratinocytes ("Kera") and fibroblasts were treated with I-BET726 (50 nм), cells were further cultured in I-BET726-containing medium for 48-72 h, cell viability $\mathbf{a}$, proliferation $\mathbf{b}, \mathbf{c}$, and cell migration $\mathbf{d}$ were tested by the appropriate assays. ${ }^{*} p<0.05$ vs. "Ctrl" group. Bar $=100 \mu \mathrm{m} \mathbf{c}$, $\mathbf{d}$.

CRISPR/Cas9 BRD4-KO plasmid was transfected to A431 cells. Following selection two stable cell lines, BRD4-KO-sL1/2, were established, showing completely depleted BRD4 expression (Fig. 4b). Caspase-3 and PARP cleavages were detected in BRD4-KO cells, indicating apoptosis activation (Fig. 4b). Importantly, in the BRD4KO A431 cells, I-BET726 (50 nM) can still induce significant cytotoxicity (MTT OD reduction, Fig. 4c), indicating the involvement of BRD4-independnent mechanism by this compound. 


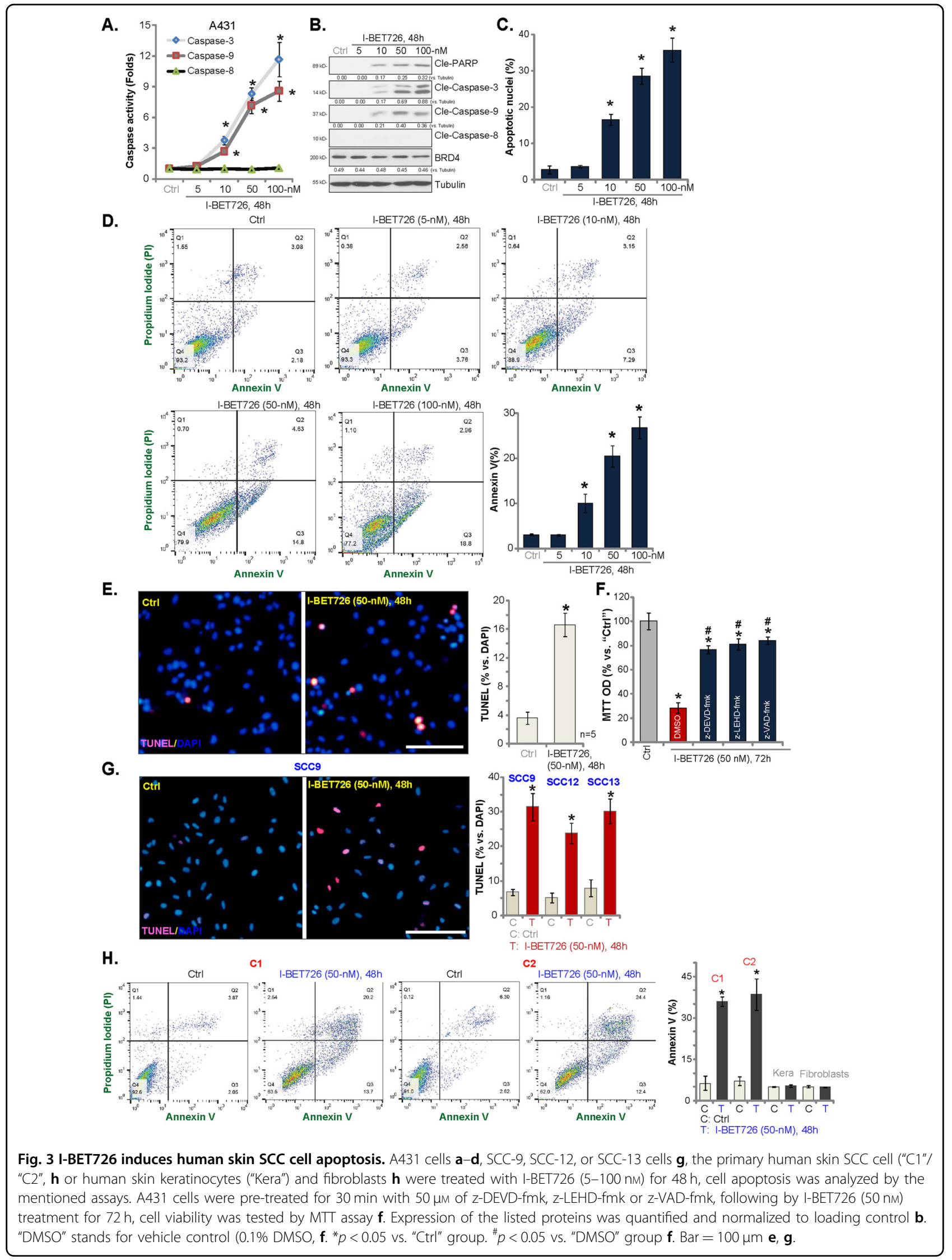




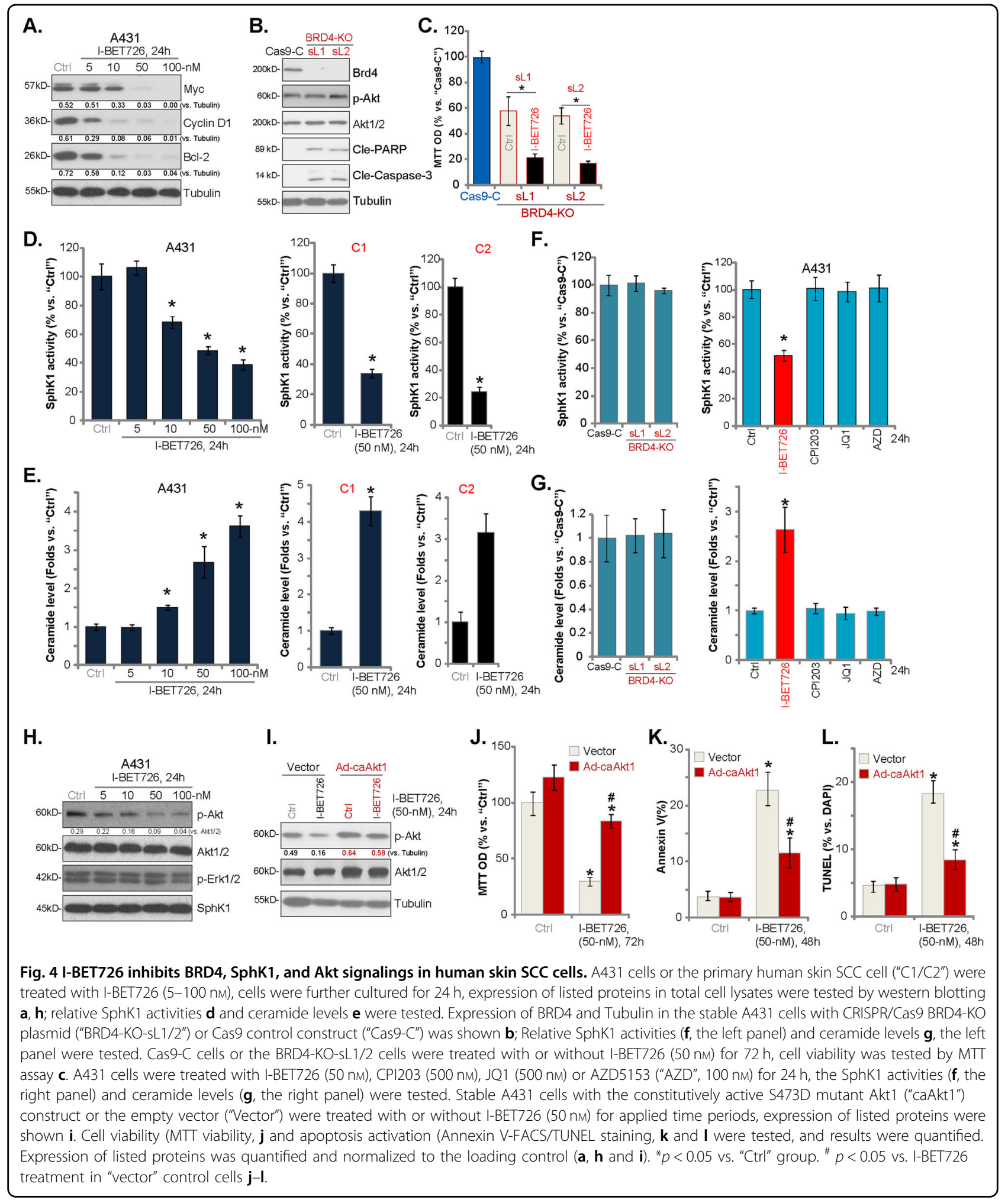

Existing studies have demonstrated a pivotal role of SphK1 in skin SCC progression ${ }^{28,29}$. SphK1 overexpression/hyperactivation promotes skin SCC cell proliferation and metastasis ${ }^{28,29}$. On the contrary, SphK1 inhibition or silencing shall lead to ceramide production and cell apoptosis ${ }^{28,29}$. SphK1 activity was therefore analyzed. We show that I-BET726 dose-dependently decreased SphK1 activity in A431 cells (Fig. 4d, left panel). 
SphK1 activity was inhibited by I-BET726 (50 nM) in the primary skin SCC cells ("C1/C2") as well (Fig. 4d, right panel). Correspondingly, the cellular ceramide levels were increased in I-BET726-treated skin SCC cells (Fig. 4e). In three other established SCC cell lines, SCC-9, SCC-12, SCC-13, I-BET726 treatment (50 nм, $24 \mathrm{~h}$ ) also induced ceramide accumulation (Fig. S2A).

Interestingly, CRISPR/Cas9-induced BRD4-KO failed to affect SphK1 activation and ceramide contents (Fig. 4f, g, the left panels). Treatment with other BRD4 inhibitors (JQ1, CPI203, and AZD5153) had no significant effect on SphK1 activation (Fig. 4f, the right panel) and ceramide production (Fig. 4g, the right panel) in A431 cells. These results suggest that SphK1 inhibition should be an unique action by I-BET726, independent of BRD4 inhibition.

Following SphK1 inhibition, accumulated ceramide could activate serine/threonine phosphatases (i.e., PP2A and PP1A) to de-phosphorylate and in-activate $\mathrm{Akt}^{30-32}$. In the present study, we show that Akt phosphorylation was inhibited by I-BET726 in A431 cells (Fig. 4h). Erk1/2 phosphorylation as well as SphK1 expression were not affected (Fig. 4h).

To test the link between Akt inhibition and I-BET726induced anti-A431 cell activity, the adenovirus-packed constitutively active S473D mutant Akt1 (caAkt1, from Dr. $\mathrm{Cao}^{18}$ ) was transduced to A431 cells, which completely restored Akt activation in I-BET726-treated cells (Fig. 4i). Importantly, in A431 cells caAkt1 largely attenuated I-BET726-induced viability reduction (Fig. 4j), and apoptosis activation (Fig. 4k, l). Therefore, Akt inhibition is involved in I-BET726-induced cytotoxicity in A431 cells.

Importantly, we show that Erk inhibition by PD98059 potentiated I-BET726-induced viability reduction (Fig. S2B) and cell death (Fig. S2C) in A549 cells. Furthermore, I-BET726 inhibited, but not blocked, Akt activation in SCC cells (Fig. 4h). LY294002, the PI3K-Akt blocker ${ }^{33}$, intensified I-BET726-induced cytotoxicity in A431 cells (Fig. S2b, c). These results suggest that Erk and PI3K-Akt activation might be the resistance mechanisms of IBET726 in SCC cells. Collectively, our results suggest that I-BET726 inhibits BRD4, SphK1, and Akt signalings in skin SCC cells.

\section{I-BET726 oral administration inhibits growth of subcutaneous A431 xenografts in SCID mice}

In order to test the activity of I-BET726 in vivo, a xenograft model was established by s.c. injection of A431 cells to the flanks of SCID mice. A431 xenograft tumors were established (close to $100 \mathrm{~mm}^{3}$ ) within 18-21 days, when I-BET726 administration was started ("Day-0"). By recording tumor volumes, we show that oral administration of I-BET726 $(5-25 \mathrm{mg} / \mathrm{kg}$ body weight, gavage, daily for 20 days) potently inhibited A431 xenograft growth in SCID mice (Fig. 5a). The estimated daily tumor growth, calculated by (tumor volume at Day35 subtracting tumor volume at Day-0)/35, was significant inhibited by I-BET726 administration (Fig. 5b). At Day35 , all tumors were individually weighted, and results show that I-BET726-treated tumors were significantly lighter than the vehicle control tumors (Fig. 5c). The animal body weights were not significantly different between three groups (Fig. 5d). Therefore, I-BET726 oral administration potently inhibited growth of subcutaneous A431 xenografts in SCID mice.

At treatment "Day-5" and "Day-10", one tumor of each group was isolated. Analyzing tumor tissue lysates by western blots demonstrated that BRD4-regulate proteins (Bcl-2, Myc, cyclin D1) were significantly downregulated in I-BET726-treated A431 tumor tissues (Fig. 5e). As compared with vehicle control tumors, Akt inactivation (Fig. 5e) and SphK1 inhibition (Fig. 5f) were also detected in I-BET726-treated tumor tissues. The levels of cleavedcaspase-3 and cleaved-PARP were increased, indicating apoptosis activation in I-BET726-treated A431 tumor tissues (Fig. 5e). Therefore, in line with in vitro findings, IBET726 oral administration inhibited BRD4, SphK1, and Akt signalings in A431 xenografts.

\section{Discussion}

BRD4 binds to the chromatin to re-activate silenced genes in the process of mitosis ${ }^{5,34}$. In addition, BRD4 phosphorylates $\mathrm{P}-\mathrm{TEFb}$ and RNA polymerase II to promote transcription elongation ${ }^{5,34}$. BRD4 is essential for the transcription of several key oncogenes, including $c-M y c, B c l-2$ and cyclin $D 1^{4,35}$. Moreover, BRD4 is important for the activation of oncogenic nuclear factor-kappa B signaling in cancer cells ${ }^{4}$. Our previous study has shown that BRD4 is overexpressed in skin SCC cells, functioning as a potential key pro-cancerous molecule $^{6}$. Targeting BRD4, i.e., by AZD5153, can potently inhibit skin SCC cell growth, in vitro and in vivo ${ }^{6}$.

In the present study, we show that I-BET726, a novel BRD4 inhibitor ${ }^{7}$, inhibited survival, proliferation, cell cycle progression, and migration in multiple established skin SCC cell lines (A431/SCC-9/SCC-12/SCC-13) and primary human skin SCC cells. I-BET726 provoked apoptosis in skin SCC cells. It was highly potent in killing skin SCC cells, more efficient than the other known BRD4 inhibitors (JQ1, CPI203, and AZD5153). Significantly, it was non-cytotoxic to normal skin keratinocytes and fibroblasts, where BRD4 levels are extremely low ${ }^{6}$. In vivo, I-BET726 oral administration inhibited A431 xenograft growth in SCID mice. Downregulation of BRD4dependent oncogenic proteins (c-Myc, Bcl-2, and cyclin D1) was detected in I-BET726-treated skin SCC cells and A431 xenografts. These results suggest that I-BET726 


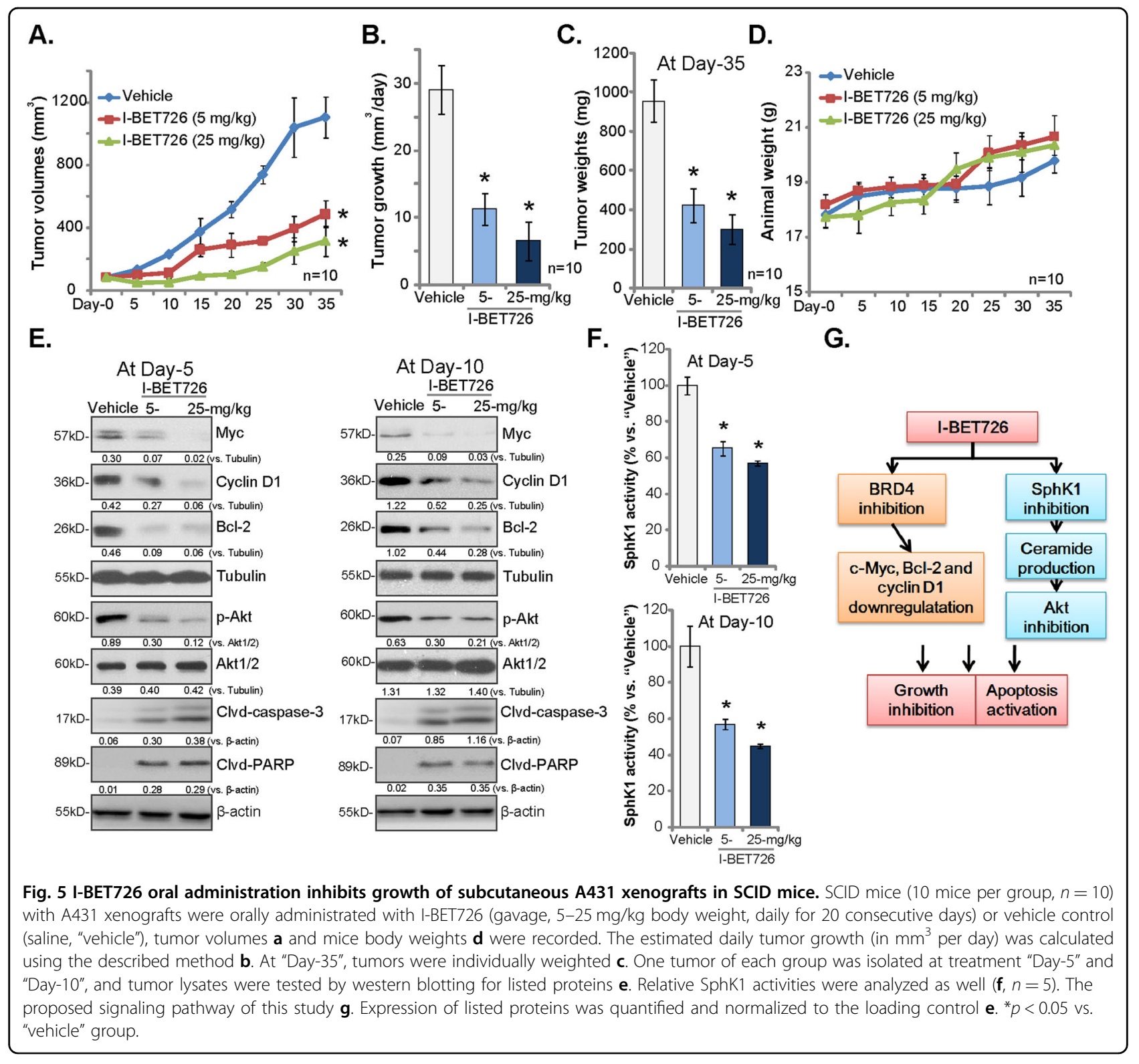

potently inhibited skin SCC cell progression in vitro and in vivo.

The outcomes for the current treatments of advanced skin SCC have been disappointing. The better skin SCC therapies should include rational inhibition of key molecular targets in multiple pro-survival/growth signalings. The facts that I-BET726 is more efficient than other known BRD4 inhibitors and it could still induce cytotoxicity in BRD4-KO A431 cells suggest the existence of BRD4-independent mechanisms by this compound. SphK1 promotes cancer cell viability, proliferation, and apoptosis resistance, as well as metastasis, and angiogenesis $^{36,37}$. Previous studies have demonstrated that SphK1 is overexpressed in skin SCC, represents as a novel prognostic marker and potential therapeutic target ${ }^{28,29}$.
The novel findings of the study are that in skin SCC cells I-BET726 can significantly inhibit SphK1 activation, followed by pro-apoptotic ceramide accumulation and Akt inactivation. These actions by I-BET726 were independent of BRD4 inhibition. As other BRD4 inhibitors (JQ1, CPI203, and AZD5153) failed to change SphK1 activity nor Akt signaling. Concurrent inhibition of BRD4, SphK1, and Akt signalings by I-BET726 could probably explain its superior anti-skin SCC activity, better than the known BRD4 inhibitors. SphK1-Akt inhibition was also detected in I-BET726treated A431 xenograft tissues. Thus, I-BET726 concurrently inhibits BRD4, SphK1, and Akt signalings in skin SCC cells (see the proposed signaling pathway of this study, Fig. 5g). 
The effects of inhibiting Akt-mTOR in combination with other cascades pathways are being tested in a number of phase I-II clinical trials ${ }^{38,39}$. These trials highlighted the importance of concurrent targeting multiple oncogenic signalings to efficiently inhibit cancer cell growth and induce apoptosis ${ }^{38,39}$. In the current study we show that I-BET726 concurrently inhibited BRD4, SphK1, and Akt signalings in established and primary skin SCC cells. This might be one reason of its superior anti-SCC cell activity. Importantly, we show that Erk inhibition or PI3K-Akt blockage potentiated I-BET726-induced cytotoxicity in SCC cells, suggesting that Erk and PI3K-Akt activation could be key resistance mechanisms of IBET726 in SCC cells. Although additional studies will be needed to further support this notion.

\section{Conclusion}

I-BET726 potently inhibits BRD4, SphK1-Akt signalings, and human skin SCC cell growth, in vitro and in vivo.

\section{Acknowledgements}

This study was supported by the National Natural Science Foundation (81773192, 81472786, 81902715, and 81774109), Foundation of Tumor Clinical and Basic Research Team of Affiliated Kunshan Hospital of Jiangsu University (KYC005), Jiangsu Province Youth Medical Talents Project (QNRC2016527), Jiangsu Province "333 Project" Research Projects (2016-III-0367); Kunshan Science and Technology Program (KS18057), Jiangsu University Clinical Medical Science and Technology Development Fund (JLY20180012), Natural Science Foundation of Jiangsu Province (BK20171248), The Scientific Research Program for Young Talents of China National Nuclear Corporation (Shang Cai), The Natural Science Foundation of Jiangsu Province (BK20180195). The funders had no role in the study design, data collection, and analysis, decision to publish, or preparation of the manuscript.

\section{Author details}

'Department of Burn and Plastic Surgery, The First Affiliated Hospital of Wenzhou Medical University, Wenzhou, China. ${ }^{2}$ Department of Radiotherapy and Oncology, Affiliated Kunshan Hospital of Jiangsu University, Kunshan, China. ${ }^{3}$ Department of Radiotherapy and Oncology, the Second Affiliated Hospital of Soochow University, Suzhou, China

\section{Conflict of interest}

The authors declare that they have no conflict of interest.

\section{Publisher's note}

Springer Nature remains neutral with regard to jurisdictional claims in published maps and institutional affiliations.

Supplementary Information accompanies this paper at (https://doi.org/ 10.1038/s41419-020-2515-z).

Received: 1 January 2020 Revised: 9 April 2020 Accepted: 14 April 2020 Published online: 05 May 2020

\section{References}

1. Walls, B., Jordan, L., Diaz, L. \& Miller, R. Targeted therapy for cutaneous oncology: a review of novel treatment options for non-melanoma skin cancer: part I. J. Drugs Dermatol. 13, 947-952 (2014).
2. Walls, B., Jordan, L., Diaz, L. \& Miller, R. Targeted therapy for cutaneous oncology: a review of novel treatment options for non-melanoma skin cancer: part II. J. Drugs Dermatol. 13, 955-958 (2014).

3. Cleavenger, J. \& Johnson, S. M. Non melanoma skin cancer review. J. Ark. Med. Soc. 110, 230-234 (2014).

4. Hajmirza, A., et al. BET Family Protein BRD4: an emerging actor in NFkappaB signaling in inflammation and cancer. Biomedicines. 6, 16 (2018).

5. $\mathrm{Fu}, \mathrm{L}$. L. et al. Inhibition of BET bromodomains as a therapeutic strategy for cancer drug discovery. Oncotarget 6, 5501-5516 (2015).xS

6. Xiang, T. et al. Bromodomain protein BRD4 promotes cell proliferation in skin squamous cell carcinoma. Cell Signal. 42, 106-113 (2018).

7. Gosmini, R. et al. The discovery of I-BET726 (GSK1324726A), a potent tetrahydroquinoline ApoA1 up-regulator and selective BET bromodomain inhibitor. J. Med. Chem. 57, 8111-8131 (2014).

8. Zou, Y., Ge, M. \& Wang, X. Targeting PI3K-AKT-mTOR by LY3023414 inhibits human skin squamous cell carcinoma cell growth in vitro and in vivo. Biochem. Biophys. Res Commun. 490, 385-392 (2017).

9. Liu, Z. et al. IGF2BP1 over-expression in skin squamous cell carcinoma cells is essential for cell growth. Biochem. Biophys. Res Commun. 501, 731-738 (2018).

10. Fan, C., Wang, W., Jin, J., Yu, Z. \& Xin, X. RASSF10 is epigenetically inactivated and suppresses cell proliferation and induces cell apoptosis by activating the p53 signalling pathway in papillary thyroid carcinoma cancer. Cell Physiol. Biochem. 41, 1229-1239 (2017).

11. Tang, C. Z. et al. Activation of Nrf2 by Ginsenoside Rh3 protects retinal pigment epithelium cells and retinal ganglion cells from UV. Free Radic. Biol. Med. 117, 238-246 (2018).

12. Chen, M. B. et al. Itraconazole-induced inhibition on human esophageal cancer cell growth requires AMPK activation. Mol. Cancer Ther. 17, 1229-1239 (2018).

13. Sun, J. et al. Galphai1 and Galphai3mediate VEGF-induced VEGFR2 endocytosis, signaling and angiogenesis. Theranostics 8, 4695-4709 (2018).

14. Wang, S. S. et al. Triptonide inhibits human nasophanyngeal carcinoma cell growth via disrupting Lnc-RNA THOR-IGF2BP1 signaling. Cancer Lett. 443, 13-24 (2019).

15. Zhang, D., Xia, H., Zhang, W. \& Fang, B. The anti-ovarian cancer activity by WYE-132, a mTORC1/2 dual inhibitor. Tumour Biol. 37, 1327-1336 (2016).

16. Yang, Y. L. et al. Sphingosine kinase-1 inhibition sensitizes curcumin-induced growth inhibition and apoptosis in ovarian cancer cells. Cancer Sci. 103, 1538-1545 (2012).

17. Gong, L. et al. Bortezomib-induced apoptosis in cultured pancreatic cancer cells is associated with ceramide production. Cancer Chemother. Pharmacol. 73, 69-77 (2014).

18. Liu, Y. Y. et al. microRNA-200a downregulation in human glioma leads to Galphai1 over-expression, Akt activation, and cell proliferation. Oncogene 37, 2890-2902 (2018)

19. Qiu, H. et al. JQ1 suppresses tumor growth through downregulating LDHA in ovarian cancer. Oncotarget 6, 6915-6930 (2015).

20. Kumar, K. et al. GLI2-dependent C-MYC upregulation mediates resistance of pancreatic cancer cells to the BET bromodomain inhibitor JQ1. Sci. Rep. 5, 9489 (2015).

21. Wong, C. et al. The bromodomain and extra-terminal inhibitor CPI203 enhances the antiproliferative effects of rapamycin on human neuroendocrine tumors. Cell Death Dis. 5, e1450 (2014).

22. Xu, K. et al. AZD5153, a novel BRD4 inhibitor, suppresses human thyroid carcinoma cell growth in vitro and in vivo. Biochem. Biophys. Res Commun. 499, 531-537 (2018).

23. Rhyasen, G. W. et al. AZD5153: a novel bivalent bet bromodomain inhibitor highly active against hematologic malignancies. Mol. Cancer Ther. 15, 2563-2574 (2016)

24. Shen, G., Jiang, M. \& Pu, J. Dual inhibition of BRD4 and PI3K by SF2523 suppresses human prostate cancer cell growth in vitro and in vivo. Biochem Biophys. Res Commun. 495, 567-573 (2018)

25. Dai, X. et al. Prostate cancer-associated SPOP mutations confer resistance to BET inhibitors through stabilization of BRD4. Nat. Med. 23, 1063-1071 (2017)

26. Zhang, L., Tong, Y., Zhang, X., Pan, M. \& Chen, S. Arsenic sulfide combined with JQ1, chemotherapy agents, or celecoxib inhibit gastric and colon cancer cell growth. Drug Des. Dev. Ther. 9, 5851-5862 (2015).

27. Riedl, S. J. \& Shi, Y. Molecular mechanisms of caspase regulation during apoptosis. Nat. Rev. Mol. Cell Biol. 5, 897-907 (2004). 
28. Kato, K., Shimasaki, M., Kato, T., Segami, N. \& Ueda, Y. Expression of sphingosine kinase-1 is associated with invasiveness and poor prognosis of oral squamous cell carcinoma. Anticancer Res. 38, 1361-1368 (2018).

29. Tamashiro, P. M., Furuya, H., Shimizu, Y., lino, K. \& Kawamori, T. The impact of sphingosine kinase-1 in head and neck cancer. Biomolecules 3, 481-513 (2013).

30. Dobrowsky, R. T., Kamibayashi, C., Mumby, M. C. \& Hannun, Y. A. Ceramide activates heterotrimeric protein phosphatase 2A. J. Biol. Chem. 268, 15523-15530 (1993)

31. Law, B. \& Rossie, S. The dimeric and catalytic subunit forms of protein phosphatase $2 \mathrm{~A}$ from rat brain are stimulated by C2-ceramide. J. Biol. Chem. 270, 12808-12813 (1995).

32. Wolff, R. A., Dobrowsky, R. T., Bielawska, A., Obeid, L. M. \& Hannun, Y. A. Role of ceramide-activated protein phosphatase in ceramide-mediated signal transduction. J. Biol. Chem. 269, 19605-19609 (1994).

33. Brunn, G. J. et al. Direct inhibition of the signaling functions of the mammalian target of rapamycin by the phosphoinositide 3-kinase inhibitors, wortmannin and LY294002. EMBO J. 15, 5256-5267 (1996).
34. Shi, J. \& Vakoc, C. R. The mechanisms behind the therapeutic activity of BET bromodomain inhibition. Mol. Cell 54, 728-736 (2014).

35. Chen, R., Yik, J. H., Lew, Q. J. \& Chao, S. H. Brd4 and HEXIM1: multiple roles in P-TEFb regulation and cancer. Biomed. Res. Int. 2014, 232870 (2014)

36. Vadas, M., Xia, P., McCaughan, G. \& Gamble, J. The role of sphingosine kinase 1 in cancer: oncogene or non-oncogene addiction? Biochim. Biophys. Acta 1781, 442-447 (2008).

37. Shida, D., Takabe, K., Kapitonov, D., Milstien, S. \& Spiegel, S. Targeting SphK1 as a new strategy against cancer. Curr. Drug Targets 9, 662-673 (2008).

38. McCubrey, J. A. et al. Ras/Raf/MEK/ERK and PI3K/PTEN/Akt/mTOR cascade inhibitors: how mutations can result in therapy resistance and how to overcome resistance. Oncotarget 3, 1068-1111 (2012).

39. Chappell, W. H. et al. Ras/Raf/MEK/ERK and PI3K/PTEN/Akt/mTOR inhibitors: rationale and importance to inhibiting these pathways in human health. Oncotarget 2, 135-164 (2011). 